Wellcome Trust spends about $£ 400$ million (US\$640 million) a year on research, and has committed roughly $£ 34$ million of this to science centres and museums in the United Kingdom, including Bristol. Why?

It is crucial to explain to the public the excitement of medical research and its potential benefits without ignoring its social impact and implications. The Wellcome Trust supports initiatives such as Explore at Bristol precisely because they aim to go beyond the traditional 'hands-on' approach to explore the wider context within which biomedical science develops.

Traditional 'hands-on' science centres barely touch modern science or biomedicine, and the social context is ignored altogether. Although hands-on exhibits are powerful learning tools, they are not necessarily the best way to tackle modern biology or the social issues raised by the human genome project, for example. Yet the general public has to be informed about the place of science in society if it is to trust science and scientists.

This task is by far the most important facing the scientific community at present and the new science centres will be crucial in this regard. There may indeed be teething troubles, not least owing to the very short timescales over which these huge projects have had to develop. But the Wellcome Trust's interest is long term, not only helping to build on the solid foundations of past innovation, but also aiming to experiment with new directions.

Laurence Smaje

The Wellcome Trust, 183 Euston Road, London NW1 2BE, UK

\section{Confidentiality is vital to bioweapons control}

Sir-Biological weapons still form part of the world's arsenal, as the work of the United Nations Special Commission (UNSCOM) in Iraq has underlined ${ }^{1}$. A weapons control system based on UN Security Council resolutions implies serious threats not just to national sovereignty but also to confidential information - an important concern in today's competitive academic and industrial environment. Since a treatybased inspection regime relies on the willingness of states to sign the treaty, they have to be certain that their confidential information will remain secure.

Current negotiations in Geneva for a protocol to strengthen the biological weapons convention ${ }^{2}$ are likely to lead to a combined reporting-inspection system. As in the chemical weapons convention ${ }^{3}$, site inspections and inclusion of non-military sites are being discussed: two elements with far-reaching consequences for confidentiality issues. In addition to classical confidentiality provisions (such as guidelines, a commission, individual secrecy agreements, and restricted access to information), the selection of appropriate triggers for countries to make declarations under the protocol should weed out threats to the loss of confidential information.

The draft protocol confines required declarations of research and development to listed agents and toxins, biological defence and maximum containment facilities. The current talks are still discussing the scope of inspections and when they should be held ${ }^{2}$ - for example, should they be random? Inspections will be based on mandates defining the purpose of the inspection, ranging from confidencebuilding, auditing and clarifying information to investigating a suspected breach of the biological weapons convention.

Until now, concerns about the risk to confidential information have not been substantiated by declarations based on the current draft protocol and several practice inspections.

The trend to incorporate private institutions can also be seen in other international agreements, such as the Convention on Access to Information, Public Participation in Decision-making and Access to Justice in Environmental Matters ${ }^{4}$ and the draft Biosafety Protocol to the Convention on Biological Diversity ${ }^{5}$.

In order to increase public and political acceptance of biological research and biotechnology, confidentiality issues and the need for more transparency will have to be brought into balance.

Johannes Rath*, Bernhard Jank $\dagger$, Otto Doblhoff-Dier $\dagger$

${ }^{*}$ Institute for Zoology, University of Vienna,

Althanstrasse 14, A-1090 Vienna, Austria

$\dagger$ Institute of Applied Microbiology,

University of Agriculture,

Nussdorfer Lände 11, A-1190 Vienna, Austria

1. Seelos, C. Nature 398, 187-188 (1999).

2. http://www.brad.ac.uk/acad/sbtwc/

3. http://www.opcw.nl/cwc/cwc-eng.htm

4. http://www.unece.org/env/europe/ppconven.htm

5. http://www.biodiv.org/biosafe/bswg6/bswg6.html\#excop

\section{Devil in the detail}

Sir - William Thompson [sic] and Lord Kelvin are credited with naming Maxwell's demon in Seth Lloyd's obituary of Rolf Landauer (Nature 400, 720; 1999). The demon, or at least an editorial gremlin, is having a little joke: William Thomson (without the 'p') and Lord Kelvin were one and the same, transmuted by act of Queen Victoria in 1892.

\section{Nicholas J. Cox}

Department of Geography, University of Durham, Durham DH1 3LE, UK

\section{Turning the tide}

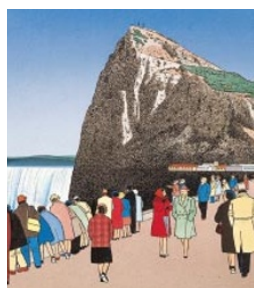

Sir - A cartoon in News and Views seems to prove that 5.33 million years ago the Mediterranean emptied into the Atlantic rather than filled from the

View from the Rock: Atlantic (Nature 400, how it should look. 613; 1999). Must we reverse our theories,

or should you reverse your slide?

Tim Robinson

Folding Landscapes, Roundstone, Connemara, Co. Galway, Ireland

\section{Medicine and biology are more than biomedicine}

Sir - While I concur with Ken Dill's call for increased support of research in physics, chemistry, mathematics and computer science, I am troubled by his reasoning ${ }^{1}$. He conflates biology and medicine into an ill-defined hybrid "biomedicine", which he believes is reducing "the problems of disease to problems of molecular science". I believe this belittles both biology and medicine.

The biological sciences are quite distinct from medicine. Obviously they overlap, but so do each of them with the other disciplines that Dill mentions. Further, advances in biology and medicine feed back into and stimulate what he terms the "basic sciences", and may lead to whole new research paradigms ${ }^{2}$.

The unidirectional model of "basic" and "applied" research implied by the pyramid in his Fig. 1 derives less from his view of their interactions than from the rapid growth of the budget of the US National Institutes of Health (which supports "biomedicine") compared with US agencies concerned with the physical sciences.

Many research programmes could influence human health and need new, long-term funding. Among these, I would stress patient-oriented medical research itself $^{3}$, including human pathophysiology, epidemiology, pharmacology, clinical trials and health-services research. A good case could also be made for behavioural, population and other as yet 'soft' sciences. If Dill wishes to present a two-dimensional model for the relationships of the sciences to the curing of disease, I would suggest a circle with radiating spokes for the many disciplines that need increased support.

Alan N. Schechter

5405 Beech Avenue, Bethesda, Maryland 20814, USA

1. Dill, K. A. Nature 400, 309-310 (1999).

2. Gershon, E. Persp. Biol. Med. 42, 95-102 (1998).

3. Goldstein, J. L. \& Brown, M. S. J. Clin. Invest. 99, 2803-2811 (1997). 